\title{
Communication
}

\section{Updates on the Mobile Divider and Its Use in Calabria Region to Monitor and Control Aethina tumida Infestation}

\author{
Camilla Di Ruggiero ${ }^{1}\left(\mathbb{D}\right.$, Ziad Mezher $^{2, *} \mathbb{C}^{\circ}$, Franco Mutinelli ${ }^{3}\left(\mathbb{D}\right.$, Alessandra De Carolis $^{1}\left(\mathbb{0}\right.$, Naomi Pocci $^{2}$ \\ and Giovanni Formato ${ }^{1}$ \\ 1 Istituto Zooprofilattico Sperimentale del Lazio e della Toscana "M. Aleandri", 00178 Rome, Italy; \\ diruggierocamilla@gmail.com (C.D.R.); decarolis.alessandra86@gmail.com (A.D.C.); \\ giovanni.formato@izslt.it (G.F.) \\ 2 Istituto Zooprofilattico Sperimentale del Lazio e della Toscana "M.Aleandri", UOT Toscana Sud-Siena, \\ 53100 Siena, Italy; naomi.pocci-esterno@izslt.it \\ 3 Istituto Zooprofilattico Sperimentale delle Venezie, NRL for Honey Bee Health, 35020 Legnaro, Italy; \\ fmutinelli@izsvenezie.it \\ * Correspondence: ziad.mezher@izslt.it; Tel.: +39-057741352
}

Citation: Di Ruggiero, C.; Mezher, Z.; Mutinelli, F.; De Carolis, A.; Pocci, N.; Formato, G. Updates on the Mobile Divider and Its Use in Calabria Region to Monitor and Control Aethina tumida Infestation. Appl. Sci. 2021, 11, 10637. https://doi.org/ 10.3390/app112210637

Academic Editor: Dimitris Mossialos

Received: 15 October 2021

Accepted: 6 November 2021

Published: 11 November 2021

Publisher's Note: MDPI stays neutral with regard to jurisdictional claims in published maps and institutional affiliations.

Copyright: (c) 2021 by the authors. Licensee MDPI, Basel, Switzerland. This article is an open access article distributed under the terms and conditions of the Creative Commons Attribution (CC BY) license (https:/ / creativecommons.org/licenses/by/ $4.0 /)$.

\begin{abstract}
The small hive beetle (SHB), Aethina tumida was first detected in the Calabria and Sicily region (Southern Italy) in 2014. In this regard, a more effective and faster inspection method than the Official one (Ministry of Health) was tested to detect the beetle in the hive. In collaboration with Calabria beekeepers' association, a "mobile divider"-based method was tested, in order to facilitate the detection of $A$. tumida and save time during hive inspection. In this short communication, we provide an update on the mobile divider technique and its related inspection procedure, which was first proposed and used in Calabria (Southern Italy) from 2014 to 2016. We report preliminary data concerning the number of detected SHBs and the time spent for their detection, based on the inspection of two apiaries in Calabria region, using both methods (official method and mobile divider method). The preliminary data presented here show that, on average, the mobile divider method seems to be able to recover a slightly higher number of beetles ( 0.9 adults) per inspected hive, in a shorter time (25 s).
\end{abstract}

Keywords: Aethina tumida; mobile divider; monitoring; control; honey bee

\section{Introduction}

The small hive beetle (SHB), Aethina tumida Murray (Coleoptera: Nitidulidae) is a parasite of sub-Saharan Africa that affects honeybee colonies. It constitutes an example of an invasive species and can have a significant impact on beekeeping [1]. In fact, both larvae and adults of this beetle live in honey bee colonies where they feed on pollen, brood and honey [2]. The larvae are also capable of infesting stored beekeeping materials such as honeycombs or combs inducing honey fermentation [1]. In September 2014, the presence of the SHB was officially confirmed in Calabria region and in November of the same year in Sicily Region (Southern Italy) [3]. Immediately after its detection, the Italian Ministry of Health established a surveillance and eradication plan [4]; diagnosis of SHB in the field was carried out by detailed clinical inspection of the colonies [5] combined with the application of internal traps and the use of sentinel colonies [6]. Eradication measures included the destruction of all colonies at the site of an infested apiary and compensation to the beekeeper $[7,8]$.

In this short communication, we provide some additional considerations about the use of the "mobile divider" adopted by beekeepers in Calabria region [9] to facilitate hive inspection in the presence of SHB. The mobile divider is placed on the opposite side from where the inspection starts and then combs of the nest are inspected one by one for the presence of SHB and transferred into an empty hive or nuc box placed close to the 
beehives under investigation. At the end of the clinical inspection, the divider is removed to check the presence of SHB behind it [9]. The official procedure to examine the hives is effective but time consuming and requires a high demand on the workforce as well as the collaboration of the beekeeper [7,9]. The mobile divider was already described as a time-saving method [9] that facilitates the detection of SHB by exploiting one of the beetle's natural behavior of escaping light [1].

In short, it provides a dark space unreachable by honey bees in which $A$. tumida hides from bee attacks and sunlight entering during hive inspections.

The mobile divider can be used in all types of hives by adapting its size to the internal dimensions of the different types of hives. It can also be installed by inexperienced beekeepers and does not require the use of any chemical compound preventing any risk of residues in bee products.

In Calabria, the local beekeepers' association (FAI Calabria) modified the original structure of the divider by coating both sides with aluminum foil. This should increase the reflection of light inside the hive and thus ease the detection of SHB while creating darker conditions behind the divider (Table 1).

Table 1. Modifications of the original structure of the mobile divider [9] carried out by beekeepers in Calabria region.

\begin{tabular}{|c|c|}
\hline MATERIALS & $\begin{array}{l}\text { The reference material is Dibond }{ }^{\circledR} \text {, the original aluminum composite sheet, comprising two } 0.3 \text { mm thick } \\
\text { aluminum cover layers and a polyethylene or mineral core. Beekeepers constructed the divider using a PVC } \\
\text { sheet coated by aluminum layers on both sides (Figure 1). One side is opaque and it is used for detecting adult } \\
\text { beetles, while the other is reflecting and is used to ease monitoring the hive's bottom. The mobile divider does } \\
\text { not increase the in-hive temperature and remains unaltered over a long time. }\end{array}$ \\
\hline SIZE & $\begin{array}{l}\text { The mobile divider thickness is few millimeters; it occupies little space and does not require any modification } \\
\text { of the internal structure of the hive. Its size is } 440 \times 350 \times 2 \mathrm{~mm} \text { according to a Dadant Blatt hive, which is the } \\
\text { standard beehive in Italy. However, the size of the mobile divider can be easily adapted to any type of hive. }\end{array}$ \\
\hline SHAPE & $\begin{array}{l}\text { The shape of the mobile divider is identical to a frame but wider than it since it needs to fit to the sides of } \\
\text { the hive. }\end{array}$ \\
\hline COSTS & The final price is about EUR 5.00. \\
\hline POSITIONING & $\begin{array}{l}\text { The bottom side of the divider remains } 2 \mathrm{~cm} \text { far from the hive floor. This allows the honey bees to access the } \\
\text { space below the divider to clean and remove } A \text {. tumida eggs or larvae if present. }\end{array}$ \\
\hline
\end{tabular}

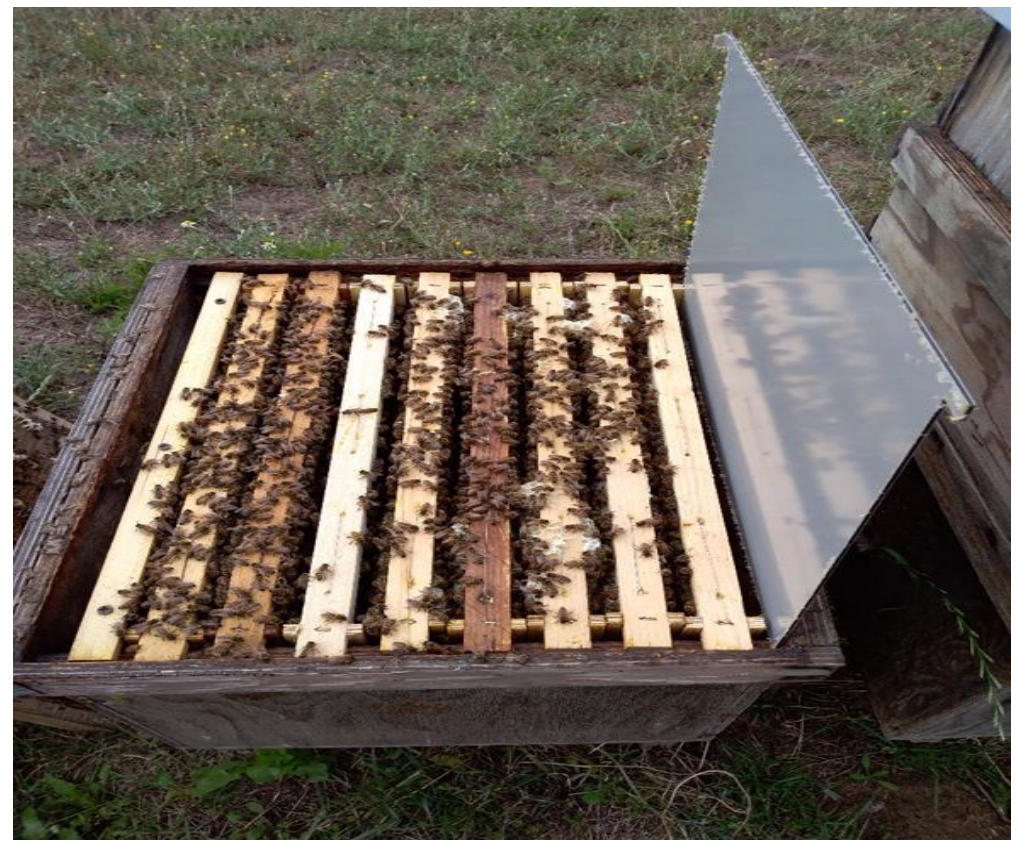

Figure 1. The mobile divider is composed of a PVC sheet coated by two aluminum layers. 


\section{Materials and Methods}

Field trials were carried out by Istituto Zooprofilattico Sperimentale del Lazio e della Toscana "M. Aleandri" (IZSLT), in collaboration with FAI Calabria in two apiaries located in SHB infested areas of the S. Ferdinando and Rizziconi districts, respectively (Calabria region) with the scope to evaluate the differences in the performance of the two methods: the official one and the one based on the mobile divider (Table 2). Accordingly, the time needed to conduct the hive inspection and the number of detected SHBs were recorded for each method in all the hives of both apiaries.

Table 2. Hive inspection procedures [9] (modified).

Official Method (Ministry of Health)
1-Remove the outer cover. Examine the external surface of the
inner cover, remove it and examine the internal surface. After
that, put the outer cover on the hive supports or on the ground.
2-To inspect the nest, remove the first lateral comb and set it
outside the hive. Then, inspect all the combs of the hive one by
one observing both surfaces after removal of the first lateral
comb. Alternatively, use an empty hive where inspected combs
could be temporarily placed.

3-Once the comb inspection is finished, return the combs to their original position.

4-If a honey super is present, examine all combs one by one. After that, remove the super and set it on the outer cover.

5-Observe the content of the bottom board if present.

\section{Mobile Divider Method (Time-Saving Protocol)}

1-An internal divider as described above should be placed between the last comb and the lateral wall of the hive.

2-Remove the outer cover. Examine the external surface of the inner cover, remove it and examine the internal surface. After that, put the outer cover on the hive supports or on the ground.

3-Then, inspect the nest starting at the first lateral comb that is on the opposite side to the divider. The inspection of the combs with pollen and honey should be more diligent, while it can be much quicker for the other brood combs.

4-Place the inspected combs one by one into an empty hive or into a nuc box.

5-In general, when combs are removed, always proceed with slow movements, in order to allow SHBs to move towards the remaining, not inspected, frames.

6-When three combs and the divider are left to be inspected, slowly move the combs to the opposite (empty) side of the hive.

7-After moving the last comb, carefully inspect the surface of the lateral divider and the space behind it, searching for the SHB. Carefully inspect also the corners, sides and bottom of the hive.

8 -If the honey super is present, remove it and inspect the surface where it was placed carefully. Then, inspect the super combs quickly and, the lateral walls more carefully.

9-Observe the content of the bottom board if present.

All hives were composed of colonies of the same strength (10 frames). Each hive was inspected twice $48 \mathrm{~h}$ apart (day 1 and 2 ) using a different protocol each day. The results obtained are presented in Tables 3 and 4 .

Table 3. Number of detected A. tumida in apiary 1 with the two different methods on day 1 and day 2.

\begin{tabular}{ccc}
\hline Apiary 1 & Day 1 & Day 2 \\
\hline ID Hive & MOBILE DIVIDER & OFFICIAL METHOD \\
\hline 1 & 2 & 2 \\
\hline 2 & 4 & 1 \\
\hline 3 & 2 & 2 \\
\hline 4 & 0 & 0 \\
\hline 5 & 0 & 1 \\
\hline
\end{tabular}


Table 3. Cont.

\begin{tabular}{|c|c|c|}
\hline Apiary 1 & Day 1 & Day 2 \\
\hline ID Hive & MOBILE DIVIDER & OFFICIAL METHOD \\
\hline 6 & $\mathbf{0}$ & $\mathbf{0}$ \\
\hline 7 & 1 & 0 \\
\hline 8 & 1 & $\mathbf{0}$ \\
\hline 9 & $\mathbf{0}$ & 1 \\
\hline 10 & $\mathbf{0}$ & $\mathbf{0}$ \\
\hline \multirow[t]{2}{*}{ Total } & 10 & 7 \\
\hline & Day 1 & Day 2 \\
\hline ID Hive & OFFICIAL METHOD & MOBILE DIVIDER \\
\hline 11 & $\mathbf{0}$ & $\mathbf{0}$ \\
\hline 12 & 0 & 0 \\
\hline 13 & 0 & 2 \\
\hline 14 & $\mathbf{0}$ & $\mathbf{0}$ \\
\hline 15 & $\mathbf{0}$ & 2 \\
\hline 16 & 0 & 1 \\
\hline 17 & 1 & 0 \\
\hline 18 & $\mathbf{0}$ & $\mathbf{0}$ \\
\hline 19 & $\mathbf{0}$ & 2 \\
\hline 20 & 1 & 3 \\
\hline Total & 2 & 10 \\
\hline
\end{tabular}

Table 4. Number of detected A. tumida in apiary 2 with the two methods on day 1 and day 2.

\begin{tabular}{|c|c|c|}
\hline Apiary 2 & Day 1 & Day 2 \\
\hline ID Hive & OFFICIAL METHOD & MOBILE DIVIDER \\
\hline 1 & 11 & 8 \\
\hline 2 & 1 & 2 \\
\hline 3 & 1 & 5 \\
\hline 4 & 8 & 10 \\
\hline 5 & $\mathbf{0}$ & 1 \\
\hline 6 & 1 & 1 \\
\hline 7 & 2 & 6 \\
\hline 8 & 8 & 3 \\
\hline 9 & 1 & 3 \\
\hline 10 & $\mathbf{0}$ & 2 \\
\hline \multirow[t]{2}{*}{ Total } & 33 & 41 \\
\hline & Day 1 & Day 2 \\
\hline ID Hive & MOBILE DIVIDER & OFFICIAL METHOD \\
\hline 11 & 8 & 5 \\
\hline 12 & 5 & 3 \\
\hline 13 & 20 & 7 \\
\hline
\end{tabular}


Table 4. Cont.

\begin{tabular}{ccc}
\hline Apiary 2 & Day 1 & Day 2 \\
\hline ID Hive & OFFICIAL METHOD & MOBILE DIVIDER \\
\hline 14 & 3 & 3 \\
\hline 15 & 2 & 2 \\
\hline 16 & 1 & 1 \\
\hline 17 & 7 & 11 \\
\hline 18 & 2 & 1 \\
\hline 19 & 8 & 5 \\
\hline 20 & 1 & 2 \\
\hline Total & 57 & 40 \\
\hline
\end{tabular}

Statistical analyses were performed using Microsoft excel spreadsheets (Microsoft ${ }^{\circledR}$, Office 2016) and R software (version 1.1.463).

\section{Results and Discussion}

In total, 40 independent observations were collected for each method. Data were aggregated and analyzed without considering the order in which the methods were applied in the apiaries because of the limited number of observations. Summary results about the duration of the inspection and the number of detected SHBs are reported in Table 5.

Table 5. Summary results of the number of $A$. tumida detected and inspection time required.

\begin{tabular}{ccccc}
\hline & \multicolumn{2}{c}{ N. of SHB } & \multicolumn{2}{c}{ Inspection Time (in minutes) } \\
\cline { 2 - 5 } & Mobile Divider & Official Method & Mobile Divider & Official Method \\
\hline min & 0 & 0 & $00: 03: 00$ & $00: 02: 50$ \\
Median & 2 & 1 & $00: 05: 12$ & $00: 06: 00$ \\
Mean & 2.95 & 2.05 & $00: 05: 37$ & $00: 06: 02$ \\
Max & 20 & 11 & $00: 10: 21$ & $00: 09: 55$ \\
\hline
\end{tabular}

We also divided the number of detected SHBs according to five class intervals in order to evaluate the trend of SHB population in each hive and the detection efficacy of each method (Figure 2).

The data obtained did not follow a normal distribution (Shapiro test; $1.779 \times 10^{-7}$ for the Mobile divider and $8.574 \times 10^{-5}$ for the Official method). Data reported in Figure 2 show that, regardless of the number of SHB present at the time of inspection, both methods were able to detect the beetles with almost identical frequency without denoting a clear relationship or pattern.

We also applied the non-parametric Wilcoxon-test to compare the results from both methods, but no statistically significant differences were detected ( $p$-value 0.1079). Apparently, only small variations concerning the number of detected beetles as well as the time needed to inspect the hives were registered where the mean number of beetles was slightly higher (2.95 vs. 2.05) and the mean inspection time for the mobile divider method was faster by $25 \mathrm{~s}$.

Further to these considerations, it should be noted that the mobile divider method is applicable throughout the year during the regular visits that beekeepers carry out in their hives. On the contrary, the "official method" appears as a "method dedicated to the search of SHB only" precluding a thorough examination of the hive directed at evaluating its correct management, i.e., stocks, health status, brood development, presence of pollen, organization of the hive, etc. 


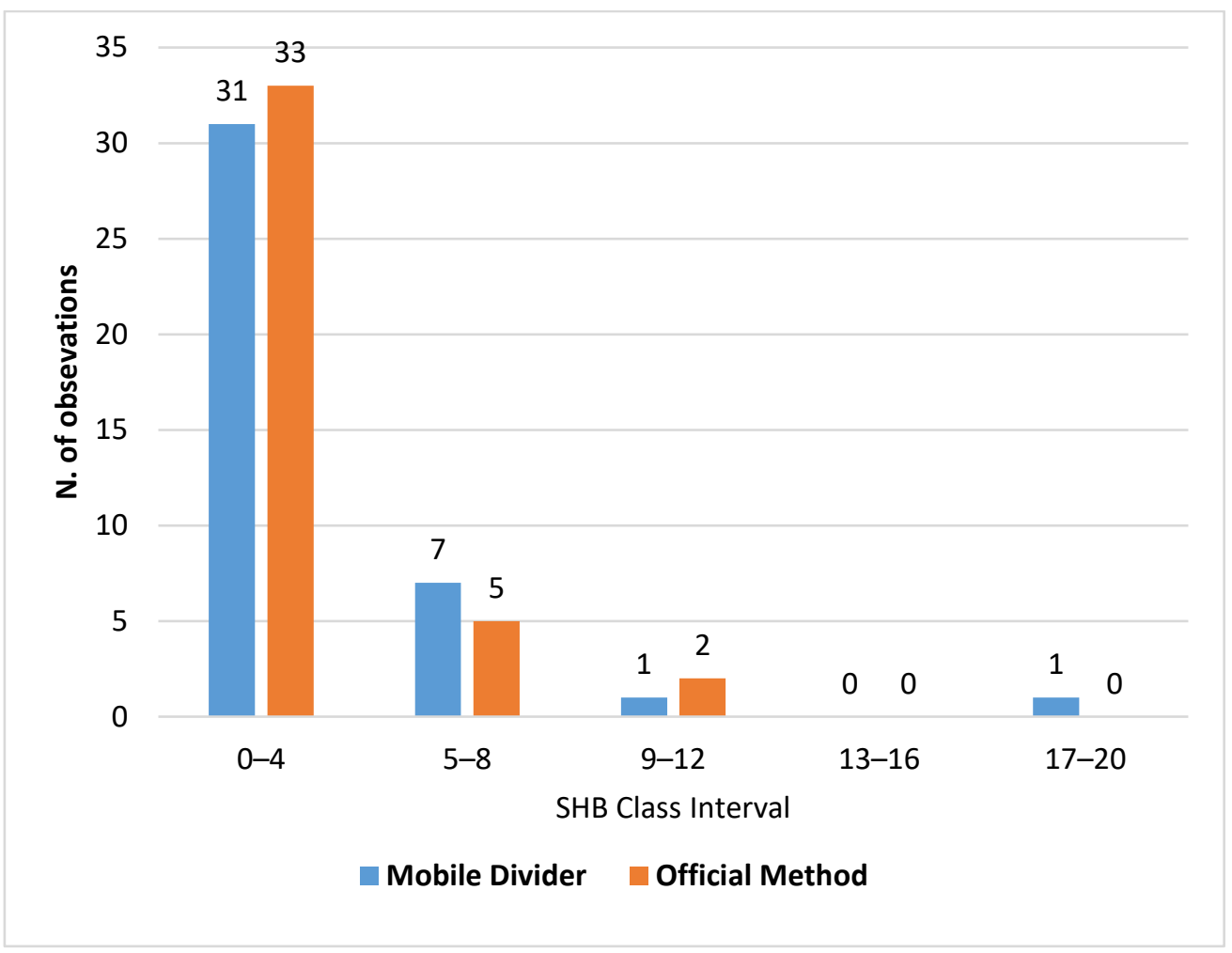

Figure 2. Number of observations per class intervals for each method.

\section{Conclusions}

This study reported preliminary data about an innovative beekeeping technique, which should be able, according to local beekeepers, to improve and ease SHB monitoring in Calabria during hive inspections. The scope was to investigate the differences between the officially recognized method for clinical inspection of the hive and the mobile divider method in terms of time saving and SHB detection efficacy. Some encouraging results were obtained but the collected data were not sufficient to allow robust statistical evaluation and further studies should be conducted on a larger honeybee colony population and under different field conditions. Unlike the "official method", the mobile divider method appears applicable all over the year during the regular visits that beekeepers carry out in their apiaries combining SHB search with a thorough examination of the hives needed to evaluate the correct development of the colonies and the appropriateness of the beekeeping management. Moreover, the mobile divider might represent a promising tool that could improve the performances of the official monitoring programs in non-infested areas, as well as a device to enhance the control of SHB infestations in the apiaries.

Author Contributions: Conceptualization, G.F., C.D.R. and A.D.C.; methodology, G.F.; validation, G.F., C.D.R. and A.D.C.; formal analysis, Z.M. and N.P.; data curation, Z.M. and N.P.; writingoriginal C.D.R. and A.D.C.; writing-review and editing, G.F., Z.M. and F.M.; supervision, G.F.; project administration, G.F. All authors have read and agreed to the published version of the manuscript.

Funding: This research received no external funding.

Institutional Review Board Statement: Not applicable.

Informed Consent Statement: Not applicable.

Data Availability Statement: Details regarding data supporting reported results can be requested to giovanni.formato@izslt.it (IZSLT).

Acknowledgments: Authors wish to thank the FAI Calabria beekeepers' association in the persons of Francesco Artese and Santo Panzera, for their technical support and active collaboration. 
Conflicts of Interest: The authors declare no conflict of interest.

\section{References}

1. Neumann, P.; Pettis, J.S.; Schäfer, M.O. Quo vadis Aethina tumida? Biology and control of small hive beetles. Apidologie 2016, 47, 427-466. [CrossRef]

2. $\quad$ Elzen, P.J.; Baxter, J.R.; Westervelt, D.; Randall, C.; Delaplane, K.S.; Cutts, L.; Wilson, W.T. Field control and biology studies of a new pest species, Aethina tumida Murray (Coleoptera, Nitidulidae), attacking European honey bees in the Western Hemisphere. Apidologie 1999, 30, 361-366. [CrossRef]

3. Mutinelli, F.; Montarsi, F.; Federico, G.; Granato, A.; Maroni Ponti, A.; Grandinetti, G.; Ferrè, N.; Franco, S.; Duquesne, V.; Rivière, M.-P.; et al. Detection of Aethina tumida Murray (Coleoptera: Nitidulidae.) in Italy: Outbreks and early reaction measures. J. Apic. Res. 2014, 53, 569-575. [CrossRef]

4. Italian Ministry of Health $(\mathrm{MoH})$. Indicazioni Sulla Attività di Sorveglianza Nelle Regioni in Cui non è Stata Rilevata la Presenza di Aethina Tumida [Indications for the Surveillance in the Regions Where Aethina Tumida Has Not Yet Been Detected] Note n. 0020069-01/10/2014-DGSAF-COD_UO-P. Available online: https://www.izsvenezie.it/documenti/temi/api/normativa/ ministero-salute/2014-10-01-nota-20069.pdf (accessed on 26 September 2021).

5. Neumann, P.; Evans, J.D.; Pettis, J.S.; Pirk, C.W.W.; Schäfer, M.O.; Tanner, G.; Ellis, J.D. Standard methods for small hive beetle research. J. Apic. Res. 2013, 52, 1-32. [CrossRef]

6. Formato, G.; Federico, G.; Di Ruggiero, C.; Pietropaoli, M.; Milito, M.; Mutinelli, F. Definition of a protocol to manage and officially confirm SHB presence in sentinel honey bee colonies. Appl. Sci. 2021, 11, 8260. [CrossRef]

7. Italian Ministry of Health $(\mathrm{MoH})$. Decreto 19 November 2014. Misure Straordinarie di Eradicazione ed Indennizzo Conseguente All'infestazione da Aethina Tumida. [Ministry of Health. Decree 19 November 2014. Extraordinary Measures of Eradication and Consequent Compensation to Aethina Tumida Infestation]. O.J. n. 294 of 19 December 2014. Available online: https: / / www.gazzettaufficiale.it/eli/id/2014/12/19/14A09715/sg (accessed on 26 September 2021).

8. Italian Ministry of Health $(\mathrm{MoH})$. Aethina Tumida-Piano di Sorveglianza Nazionale-Anno 2021 [Aethina Tumida-National Surveillance Program-Year 2021]. Note n. 0009266-13/04/2021-DGSAF-MDS-P. Available online: https:/ / www.izsvenezie.it/ documenti/temi/api/normativa/ministero-salute/2021-04-13-nota-9966.pdf (accessed on 26 September 2021).

9. Rivera-Gomis, J.; Gregorc, A.; Maroni Ponti, A.; Artese, F.; Zowitsky, G.; Formato, G. Monitoring of small hive beetle (Aethina Tumida Murray) in Calabria (Italy) from 2014 to 2016: Practical identification methods. J. Apic. Sci. 2017, 61, 257. [CrossRef] 